\title{
Optical Clarity and Cataract: The Contributions of Intermediate Filaments to the Biology of the Ocular Lens
}

\author{
Paul FitzGerald ${ }^{1 *}$, Brad Shibata ${ }^{1}$ and Ning Sun ${ }^{1}$ \\ 1. Dept. of Cell Biology and Human Anatomy, School of Medicine, University of California, Davis, CA \\ USA. \\ * Corresponding author: pgfitzgerald@ucdavis.edu
}

The lens of the eye operates under biological constraints that do not apply to most tissues: it must be optically clear, have a very high index of refraction so it can bend light, it must have a precise focal point and lack spherical aberration, and finally it must be elastically deformable in order to alter the focal point between near and far objects.

Our work has sought to establish the biological adaptations that permit the lens to meet these requirements. We have focused our efforts on a cytoskeletal filament referred to as the Beaded Filament (BF) [1]. The $\mathrm{BF}$ is distinct from thin filaments and intermediate filaments (IFs), and has been demonstrated only in the terminally-differentiated fiber cells of the lens, cells which make up about $95 \%$ of the mass of the lens [2].

Subcellular fractionation and SDS PAGE analysis implicated two lens-specific proteins, CP49 (bfsp2) and filensin (bfsp1), as candidates for BF proteins. Electron microscope level immunocytochemistry with monoclonal antibodies to both proteins established that both were components of the BF [3]. Targeted gene disruption was used to generate both CP49 and filensin knockout mice. Electron microscopy of the knockout animals established that in the absence of either protein, BFs did not assemble, confirming that both proteins were required for BF assembly [4]. DNA sequencing and determination of gene structure established that both CP49 and filensin were members of the large IF family of proteins [5-7]. However, sequence analysis established that these were the most divergent members of the IF family. For this reason the BF proteins are the founding (and only) members of a new class of IF proteins referred to variably as either "orphan filaments" or Type VI Ifs [8]. Notably, these are the only two IF proteins which assemble into something other than the canonical 10-11 nm IF. In all vertebrate lenses, fiber cells are arranged with an astounding precision. This feature, because of its conservation and uniqueness, has been considered essential for optical clarity [9]. Scanning EM of the lenses from KO mice showed that fiber cells could achieve the highly elaborate cellular and tissue level phenotype, but that they were unable to maintain that phenotype with age [9]. In the KO mice, the fiber cell architecture disintegrated into a randomization of fiber cell and lens structure, though not in fiber cell death. This proved to be the first test of the hypothesis that this long-range order of fiber cells was essential to clarity. Surprisingly, the KO mice showed no loss of lens clarity until about three months of age, at which time a very subtle light scatter was barely detectable, a light scatter that never rose to the level of cataract [10]. This contrasts with an inherited human cataract whose cause was traced to a point mutation in bfsp2, and which resulted in an adolescent onset cataract requiring extraction [11,12]. Finally, mechanical assessment of the lens elastic properties showed that lenses from the KO mice exhibited lower stiffness and an unexpected higher resilience than the wild-type lenses. The KO lenses were smaller, and had a higher ratio of axial:equatorial diameter [13]. These data demonstrate that several of the unique requirement for the lens are dependent upon the assembly of the lens-specific BF.

Lens structure and composition introduce challenges to analysis by microscopy. Lenses continue to grow 
throughout life by addition of new layers at its surface. This means that fiber cells are arranged in exact chronological order, with the older cells at the center. Thus, there is a gradient of maturation from surface to center. In order to elevate the index of refraction of the lens, fiber cell differentiation includes an accumulation of cytoplasmic protein to very high levels. The density of protein creates a barrier to penetration of lenses fixed by immersion [14]. This means that there is a superimposition of a differentiation gradient and a fixation gradient which complicates efforts to demonstrate age/differentiation-dependent changes in cells. Further the very high density of cytoplasmic protein completely obscures the underlying cytoskeleton, precluding analysis by conventional EM.

We have adapted variants of freeze substitution and cellular "ghosting" to develop workarounds that permit us to employ both light and electron microscopy to analyses of the lens, and the testing of hypotheses of BF function $[4,15]$.

\section{References:}

[1] H Maisel and MM Perry, Exp Eye Res 14(1) (1972), p. 7.

[2] PG FitzGerald and J Casselman, Curr Eye Res 10(5) (1991), p. 471.

[3] PG FitzGerald and W Gottlieb, Curr Eye Res 8(8) (1989), p. 801.

[4] PG FitzGerald, Curr Eye Res 9(11) (1990), p. 1083.

[5] JF Hess, JT Casselman and PG FitzGerald, Curr Eye Res 12(1) (1993), p. 77.

[6] JF Hess, JT Casselman and PG FitzGerald, GJ Biol Chem 271(12) (1996), p. 6729.

[7] JF Hess et al., Exp Eye Res 66(5) (1998), p. 625.

[8] Lodish et al., in "Molecular Biology of the Cell”, S. Tenney, Editor. (Freeman, New York NY) p. 837.

[9] KH Yoon et al., Invest Ophthalmol Vis Sci 49(3) (2008), p. 1030.

[10] A Alizadeh et al., Invest Ophthalmol Vis Sci 43(12) (2002), p. 3722.

[11] YP Conley et al., Am J Hum Genet 66(4) (2000), p. 1426.

[12] PM Jakobs et al., Am J Hum Genet 66(4) (2000), p. 1432.

[13] DS Fudge et al., Invest Ophthalmol Vis Sci 52(6) (2011), p. 3860.

[14] T Blankenship et al., Invest Ophthalmol Vis Sci 48(7) (2007), p. 3269.

[15] P FitzGerald et al., Mol Vis 22 (2016), p. 970.
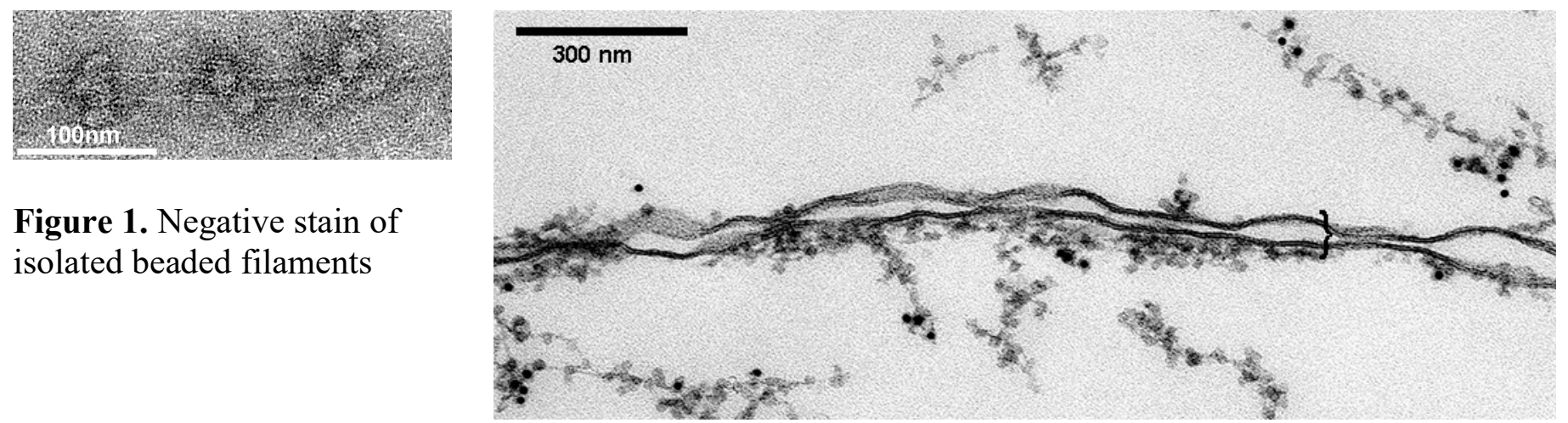

Figure 1. Negative stain of isolated beaded filaments

Figure 2. Immunocytochemical localization of filensin in "ghosts" of lens fiber cells. All filamentous structures are BFs. The plasma membrane of two fiber cells are indicated at the bracket (\}$)$ 